\title{
Experimental Results on Heavy Quarks
}

\author{
A. Stocchi ${ }^{\mathrm{a}}$ \\ ${ }^{a}$ Laboratoire de l'Accélerateur Linéaire, \\ IN2P3-CNRS et Université de Paris-Sud, BP34, F-91898 Orsay Cedex, France
}

This paper reviews the results presented at the $31^{\text {st }}$ ICHEP on Heavy Quarks, with emphasis on those related to the determination of the Unitarity Triangle parameters.

\section{Introduction}

Accurate studies of the production and decays of beauty and charm hadrons are exploiting a unique laboratory for testing the Standard Model in the fermion sector, for studying QCD in the non-perturbative regime and for searching for New Physics through virtual processes. The first two items are the main subjects of this paper while the latter is discussed in [1].

In the Standard Model, weak interactions among quarks are encoded in a $3 \times 3$ unitary matrix: the CKM matrix. The existence of this matrix conveys the fact that quarks are a linear combination of mass eigenstates [2, 3].

The CKM matrix can be parametrized in terms of four free parameters. These parameters can be measured in several physics processes.

In a frequently used parametrization these parameters are named: $\lambda, \mathrm{A}, \bar{\rho}$ and $\bar{\eta}$. The Standard Model predicts relations between the different processes which depend upon these parameters; CP violation is accommodated in the CKM matrix and its existence is related to $\bar{\eta} \neq 0$. The unitarity of the CKM matrix can be visualized as a triangle in the $\bar{\rho}-\bar{\eta}$ plane. Several quantities, depending upon $\bar{\rho}$ and $\bar{\eta}$ can be measured and they must define compatible values for the two parameters, if the Standard Model is the correct description of these phenomena. Extensions of the Standard Model can provide different predictions for the position of the upper vertex of the triangle, given by the $\bar{\rho}$ and $\bar{\eta}$ coordinates.

$\overline{1_{\bar{\rho}}=\rho\left(1-\frac{\lambda^{2}}{2}\right) \quad ; \quad \bar{\eta}}=\eta\left(1-\frac{\lambda^{2}}{2}\right)[\bar{Q}$
The most precise determination of these parameters is obtained using $\mathrm{B}$ decays and $B^{0}-\bar{B}^{0}$ oscillations.

Many additional measurements on B and D mesons properties (masses, branching fractions, lifetimes...) are necessary to constrain the Heavy Quark theories (Operator Product Expansion (OPE) / Heavy Quark Effective Theory (HQET) / Lattice QCD (LQCD)) to allow for precise extraction of the CKM parameters.

Figure 1 shows "pictorially" the unitarity triangle and the different measurements contributing to the determination of its parameters.

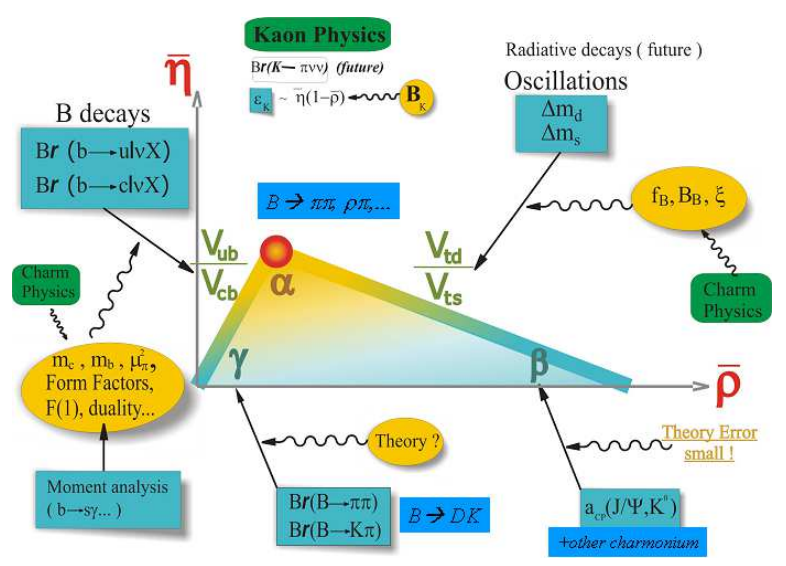

Figure 1. The unitarity triangle.

In the first part of this paper we present the 
Table 1

Summary of the main characteristics of the different facilities performing $b$-physics studies.

\begin{tabular}{|c|c|c|c|c|}
\hline Experiments & Number of $b \bar{b}$ events & Environment & Characteristics & Status \\
\hline$\overline{\mathrm{LEP}}$ & $\sim 1 M$ per expt. & $\begin{array}{l}\mathrm{Z}^{0} \text { decays } \\
\left(\sigma_{-} \sim 6 \mathrm{nb}\right)\end{array}$ & $\begin{array}{l}\text { back-to-back } 45 \mathrm{GeV} \text { b-jets } \\
\text { all B hadrons produced }\end{array}$ & Stopped \\
\hline SLD & $\sim 0.1 M$ & $\mathrm{Z}^{0}$ decays & $\begin{array}{l}\text { back-to-back } 45 \mathrm{GeV} \text { b-jets } \\
\text { all B hadrons produced } \\
\text { (beam polarized) }\end{array}$ & Stopped \\
\hline CLEO & $\sim 9 M$ & $\begin{array}{l}\Upsilon(4 S) \text { decays } \\
\left(\sigma_{b \bar{b}} \sim 1.2 \mathrm{nb}\right)\end{array}$ & $\begin{array}{l}\text { mesons produced at rest } \\
\left(B_{d}^{0} \text { and } B^{+}\right)\end{array}$ & $\begin{array}{l}\text { Running } \\
\text { (lower energies) }\end{array}$ \\
\hline BaBar & $\sim 90 M$ & $\Upsilon(4 S)$ decays & $\begin{array}{l}\text { Asymmetric B factory } \\
\left(B_{d}^{0} \text { and } B^{+}\right)\end{array}$ & Running \\
\hline Belle & $\sim 90 M$ & $\Upsilon(4 S)$ decays & $\begin{array}{l}\text { Asymmetric B factory } \\
\left(B_{d}^{0} \text { and } B^{+}\right)\end{array}$ & Running \\
\hline $\mathrm{CDF}$ & $\sim$ several $\mathrm{M}$ & $\begin{array}{l}p \bar{p} \text { collider } \\
\sqrt{s}=1.8 \mathrm{TeV}\end{array}$ & $\begin{array}{l}\text { events triggered with } \\
\text { leptons or offset tracks } \\
\text { all B hadrons produced }\end{array}$ & $\begin{array}{l}\text { Running } \\
\text { (Run II) }\end{array}$ \\
\hline
\end{tabular}

new results on the beauty and charm meson spectroscopy and lifetimes. The second part summarises the new results obtained in rare B decays. Part of these results, especially those concerning the new determination of $\sin 2 \beta$ are

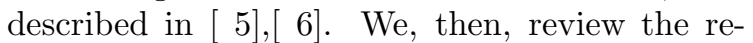
sults on the CKM matrix elements: $V_{c b}$ and $V_{u b}$ through B decays and $V_{t d}$ and $V_{t s}$ using $B^{0}-\bar{B}^{0}$ oscillations. We finally show how these measurements constrain the Standard Model in the fermion sector, through the determination of the unitarity triangle parameters.

B physics is studied at several facilities, which are schematically summarised in Table 1 .

For D physics, at the $\Upsilon(4 S)$ charm particles are produced in the continuum allowing B-factories to obtain charm physics results. Charm particles are also produced in photon and hadron production. FOCUS experiment (E831) (the successor of E687) is designed to study charm particles produced by $\simeq 200 \mathrm{GeV}$ photons using a fixed target spectrometer. SELEX experiment uses, instead, the $600 \mathrm{GeV}$ Fermilab Hyperon beam (which in fact has equal fluxes of $\pi^{-}$and $\Sigma^{-}$).

\section{Spectroscopy}

\subsection{B Spectroscopy}

New results were presented by the DELPHI Coll. on orbitally excited B mesons $\left(\mathrm{L}=1, B_{u, d, s}^{* *}\right)$ and excited B hadrons $\left(\Sigma_{b}^{(*)}\right)$.

$B_{u, d}^{* *}$ mesons.

HQET treats heavy quarks as static colour sources and the light degrees of freedom are decoupled from the heavy quark spin. The orbitally excited states can be grouped into doublets of $j_{q}$ $\left(j_{q}=s_{q}+l ; s_{q}\right.$ is the spin of the light quark and $l$ is its angular momentum relative to the heavy quark). The states with $j_{q}=1 / 2(3 / 2)$ have a broad(narrow) width, respectively.

Previous results were obtained by DELPHI, OPAL and ALEPH [ []. In these analyses it was not possible to separate the various contributions to the $B^{* *}$ signals and the more plausible hypothesis was that both narrow and broad states contributed. In the new results, from DELPHI, the dependence from the Monte Carlo background modelling, which was a critical point in the old analyses, is reduced by using purer samples and by fitting the background contribution directly on data.

The $B_{u, d}^{* *}$ mesons are reconstructed, inclusively, by combining the 4-momentum of the $\mathrm{B}$ system 

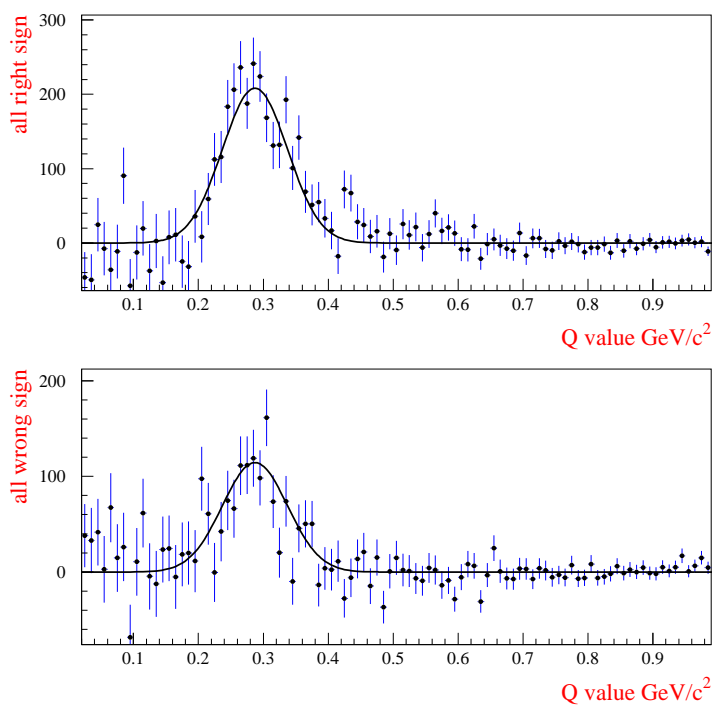

Figure 2. $\quad B_{u, d}^{* *}$ analysis. The plot, from the DELPHI Coll., shows the distribution of $Q=$ $[m(B \pi)-m(B)]$ fitted with a single Gaussian. Results of this fit are given in equation 11. All the results are preliminary.

with a charged pion having a trajectory compatible with the primary vertex position. The distribution of the mass difference $Q=[m(B \pi)-$ $m(B)]$ is shown in Figure 2. The fit is compatible with a single Gaussian distribution of width corresponding to the experimental resolution, which suggests a low mass splitting between the narrow states. The results are:

$Q=298 \pm 4 \pm 12 \mathrm{MeV} ; \sigma(Q)=47 \pm 3 \pm 5 \mathrm{MeV}$

$\frac{\sigma\left(B_{u, d}^{* *}\right)}{\sigma_{b}}=(9.8 \pm 0.7 \pm 1.2) \%($ narrow states only $)$

The data also suggest (improvement of the $\chi^{2}$ of the fit) the presence of a broad component, situated $100 \mathrm{MeV}$ above the fitted narrow states component, with a width of $\Gamma \simeq 250 \mathrm{MeV}$.

$B_{s}^{* *}$ mesons and $\Sigma_{b}^{(*)}$ baryons

Signals from $B_{s}^{* *}$ mesons can be obtained by replacing the $\pi$ candidate by an identified charged kaon. Evidence for the narrow $B_{s}^{* *}$ mesons was found by OPAL and in a preliminary DELPHI analysis [ [8]. The new DELPHI analysis does not confirm this result and sets a limit on the production rate of narrow $B_{s}^{* *}$ states:

$$
\begin{array}{r}
\frac{\sigma\left(B_{s}^{* *}\right)}{\sigma_{b}}<1.5 \% \text { at } 95 \% \text { C.L. } \\
\text { (narrow states only) }
\end{array}
$$

Excited b-baryons states are the $\Sigma_{b}(I=1, S=$ $1 / 2)$ and $\Sigma_{b}^{*}(I=1, S=3 / 2)$ in which the light diquark (ud) system has a spin and an isospin equal to one. These baryons are expected to cascade into $\Lambda_{b}^{0} \pi$. The $\Lambda_{b}^{0}$ candidate is inclusively reconstructed, as in previous analyses.

The new DELPHI analysis does not confirm an old preliminary evidence [9] by setting a limit on the production rate:

$$
\frac{\sigma\left(\Sigma_{b}^{(*)}\right)}{\sigma_{b}}<1.5 \% \quad \text { at } 95 \% \text { C.L. }
$$

The Tevatron (Run II) is in a good position to obtain signals for these states, in future.

\subsection{Charmed Baryon Spectroscopy}

22 charmed baryon states were found sofar, implying a rich spectroscopy.

New results were presented concerning the mass measurement of the $\Omega_{c}(c s s)$ baryon. The invariant mass obtained by the FOCUS Coll. is shown in Figure 3. A summary of results on the $\Omega_{c}$ mass measurement is given in Table 2 .

Table 2

Summary of $\Omega_{c}$ mass measurements.

\begin{tabular}{lll} 
Coll. & $\mathrm{M}\left(\Omega_{c}\right)[\mathrm{MeV}]$ & decay modes \\
\hline FOCUS & $2697.0 \pm 2.2$ & $\Omega^{-} \pi^{+}-\Xi K \pi \pi$ \\
Belle & $2693.7 \pm 1.3 \pm 1.1$ & $\Omega^{-} \pi^{+}$ \\
CLEO & $2694.6 \pm 2.6 \pm 1.9$ & 4 decays modes \\
\hline Average & $2694.9 \pm 1.3$ & \\
\hline
\end{tabular}

The SELEX Coll. finds signals corresponding to a possible first observation of doublecharm baryons $(\mathrm{J}=1 / 2$ ground state iso-doublet): $\Xi_{c c}^{+}(c c d) \rightarrow \Lambda_{c}^{+} K^{-} \pi^{+}$and $\Xi_{c c}^{++}(c c u) \rightarrow$ 


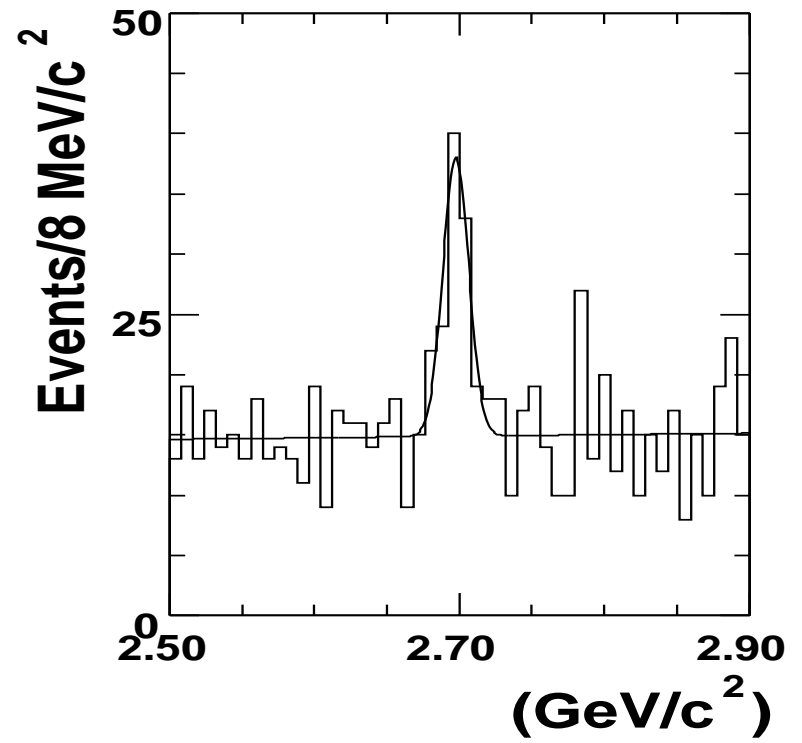

Figure 3. The invariant mass spectrum of $\Omega_{c}$ candidates as obtained by the FOCUS Coll. using $\Omega^{-} \pi^{+}$and $\Xi K \pi \pi$ decay modes. The result is preliminary.

$\Lambda_{c}^{+} K^{-} \pi^{+} \pi^{+}$at a mass of about $3520 \mathrm{MeV}$ and $3460 \mathrm{MeV}$ respectively.

This evidence is not confirmed by a similar search made by the FOCUS Coll.

\section{Heavy quark lifetimes}

The measurements of the B and D lifetimes test the decay dynamics, giving important information on non-perturbative QCD corrections induced by the spectator quark (or diquark). Decay rates are expressed using the OPE formalism, as a sum of operators developed in series of order $O\left(\Lambda_{Q C D} / m_{Q}\right)^{n}$. In this formalism, no term on $1 / m_{Q}$ is present and the spectator effects contribute at order $1 / m_{Q}^{3}$ 2. In the $\mathrm{B}$ sector, nonperturbative operators are evaluated, most reliably, using lattice QCD calculations.

\footnotetext{
${ }^{2}$ Terms at order $1 / m_{Q}$ would appear if in this expansion the mass of the heavy hadron was used instead of the mass of the quark. The presence of this term would violate the quark-hadron duality.
}

\subsection{Beauty hadron lifetimes}

Measurements of the different B hadron lifetimes have been a field of intense experimental activity at LEP/SLD/CDF in the last ten years and recently at $\mathrm{B}$-factories (for $B_{d}^{0}$ and $B^{+}$mesons only). Results are given in Table 3 [ [10].

Table 3

Summary of B hadron lifetime results (as calculated by the Lifetime Working Group [10]).

\begin{tabular}{ccc} 
B Hadrons & \multicolumn{2}{c}{ Lifetime $[\mathrm{ps}]$} \\
\hline$\tau\left(B_{d}^{0}\right)$ & $1.540 \pm 0.014$ & $(0.9 \%)$ \\
$\tau\left(B^{+}\right)$ & $1.656 \pm 0.014$ & $(0.8 \%)$ \\
$\tau\left(B_{s}^{0}\right)$ & $1.461 \pm 0.057$ & $(3.9 \%)$ \\
$\tau\left(\Lambda_{b}^{0}\right)$ & $1.208 \pm 0.051$ & $(4.2 \%)$ \\
\hline$\tau\left(B_{d}^{0}\right) / \tau\left(B^{+}\right) \quad=$ & $1.073 \pm 0.014$ \\
$\tau\left(B_{d}^{0}\right) / \tau\left(B_{s}^{0}\right) \quad=$ & $0.949 \pm 0.038$ \\
$\tau\left(\Lambda_{b}^{0}\right) / \tau\left(B_{d}^{0}\right) \quad=$ & $0.798 \pm 0.052$ \\
$\tau(\mathrm{b}-$ baryon $) / \tau\left(B_{d}^{0}\right)$ & $=0.784 \pm 0.034$ \\
\hline
\end{tabular}

Ratios of different B hadron lifetimes, given in Figure 1, are compared with theory predictions (yellow bands).

The attained experimental precision is remarkable and LEP results are still dominating the scene. The fact that charged B mesons live longer than neutral B mesons is now established at $5 \sigma$ level and is in agreement with theory. The $B_{d}^{0}$ and $B_{s}^{0}$ lifetimes are expected (at $\simeq 1 \%$ ) and found (at $\simeq 4 \%$ ) to be equal. A significant measurement in which this ratio differs from unity will have major consequences for the theory. The b-baryons lifetime is measured to be shorter than the $B_{d}^{0}$ lifetime, and the size of this effect seems to be more important than predicted $(2-3 \sigma)$. Recent calculations of high order terms give an evaluation of the b-baryon lifetime in better agreement with the experimental result [11.

New results are expected from B-Factories (which could decrease the relative error on the lifetimes of the $B_{d}^{0}$ and $B^{+}$to $0.4-0.5 \%$ ) and mainly from Tevatron (Run II) which could precisely measure all $\mathrm{B}$ hadron lifetimes, including the $\Xi_{b}, \Omega_{b}$ and the $B_{c}$. 


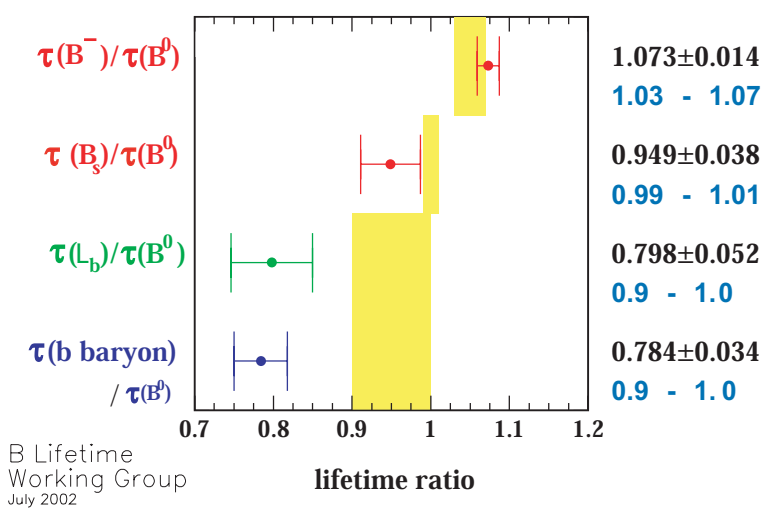

Figure 4. B hadrons lifetime ratios [ 10], compared with theoretical predictions as given by the yellow bands.

\subsection{Charm hadron lifetimes}

Differences between charm-hadron lifetimes are expected to be larger than for b-hadrons due to the smaller value of the charm quark mass.

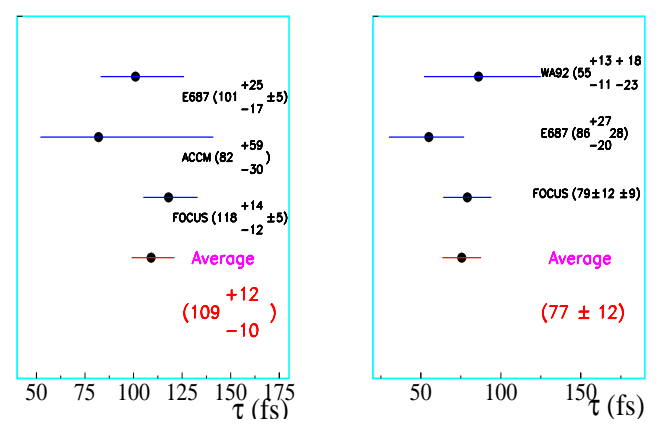

Figure 5. Summary of individual $\Xi_{c}^{0}$ and $\Omega_{c}$ charm baryon lifetimes measurements.

Important improvements have been recently made in this sector, mainly by the FOCUS Coll.,
Table 4

Summary of charm hadron lifetime results. When new results were presented at this Conference, averages have been made by the author.

\begin{tabular}{|c|c|c|}
\hline Hadrons & Lifetimes $[\mathrm{fs}]$ & Comments \\
\hline$\overline{\tau\left(D^{0}\right)}$ & $411.3 \pm 1.3$ & New Belle/FOCUS \\
\hline$\tau\left(D^{+}\right)$ & $1039.4 \pm 6.3$ & New Belle/FOCUS \\
\hline$\tau\left(D_{s}\right)$ & $490 \pm 9$ & PDG2002 \\
\hline$\tau\left(\Lambda_{c}\right)$ & $200 \pm 6$ & PDG2002 \\
\hline$\tau\left(\Xi_{c}^{+}\right)$ & $442 \pm 26$ & PDG2002 \\
\hline$\tau\left(\Xi_{c}^{0}\right)$ & $109_{-10}^{+12}$ & New FOCUS \\
\hline \multirow[t]{6}{*}{$\tau\left(\Omega_{c}\right)$} & $77 \pm 12$ & New FOCUS \\
\hline & $\overline{\tau\left(D^{+}\right) / \tau\left(D^{0}\right)}$ & $=2.53 \pm 0.02$ \\
\hline & $\tau\left(D_{s}\right) / \tau\left(D^{0}\right)$ & $=1.19 \pm 0.02$ \\
\hline & $\tau\left(\Lambda_{c}\right) / \tau\left(D^{0}\right)$ & $=0.49 \pm 0.01$ \\
\hline & $\tau\left(\Xi_{c}^{+}\right) / \tau\left(\Lambda_{c}\right)$ & $=2.21 \pm 0.15$ \\
\hline & $\tau\left(\Omega_{c}\right) / \tau\left(\Xi_{c}^{0}\right)$ & $=0.71 \pm 0.13$ \\
\hline
\end{tabular}

producing results which are often better than previous world averages. New results obtained in the baryon sector are shown in Figure 5 . A summary of charm hadron lifetime measurements is given in Table $⿴$.

The charm hadron lifetime hierarchy is observed as predicted by theory. Nevertheless, the remarkable improvement in the experimental precision is not yet matched by theory calculations.

\section{Rare B decays}

Rare B decays were the realm of CLEO Coll., with about $9 \mathrm{M}$ pairs of $\mathrm{B}$ mesons registered, which allowed to access B decay modes of branching fraction of the order of $10^{-5}$. In few areas the LEP experiments contributed too. Since the statistics is the main issue, these studies have become a central topic in the B-factory program, which have now (with about 90M pairs of $\mathrm{B}$ mesons registered) the possibility of accessing branching fractions of the order of $10^{-6}$.

\subsection{Radiative B decays ( $b \rightarrow s \gamma$ )}

The radiative $B$ decays proceed via the penguin diagrams. The first observation of these events was made by the CLEO Coll. in 1993 [ 12 .

There are three main motivations for studying radiative $\mathrm{B}$ decays: 
Table 5

Summary of the results on exclusive $b \rightarrow s \gamma$ decays. Part of these results are still preliminary and the averages have been made by the author.

\begin{tabular}{|c|c|c|}
\hline Collaboration & $B^{0} \rightarrow K^{* 0} \gamma\left[10^{-6}\right] \quad B^{-} \rightarrow K^{*-} \gamma\left[10^{-6}\right]$ & $B^{0} \rightarrow K_{2}^{* 0}(1430) \gamma\left[10^{-6}\right]$ \\
\hline$\overline{\mathrm{CLEO}}$ & $\begin{array}{c}45.5 \pm 7.0 \pm 3.4 \\
A 7.6 \pm 8.6 \pm 2.8\end{array}$ & $16.6 \pm 5.6 \pm 1.3$ \\
\hline BaBar (22.7 MB) & $\begin{array}{c}42.3 \pm 4.0 \pm 2.2 \quad 38.3 \pm 6.2 \pm 2.2 \\
A_{C P}=-0.044 \pm 0.076 \pm 0.012\end{array}$ & \\
\hline Belle (65.4 MB) & $\begin{array}{c}39.1 \pm 2.3 \pm 2.5 \quad 42.1 \pm 3.5 \pm 3.1 \\
A_{C P}=-0.022 \pm 0.048 \pm 0.017\end{array}$ & $15_{-5}^{+6} \pm 1$ (with only $\left.30 \mathrm{MB}\right)$ \\
\hline Average & $\begin{array}{cc}41.4 \pm 2.6 & 39.8 \pm 3.5 \\
A_{C P}=-0.02 \pm 0.04\end{array}$ & $16.1 \pm 4.2$ \\
\hline theory [|13],,[14],[15] & Br [40-100] & \\
\hline
\end{tabular}

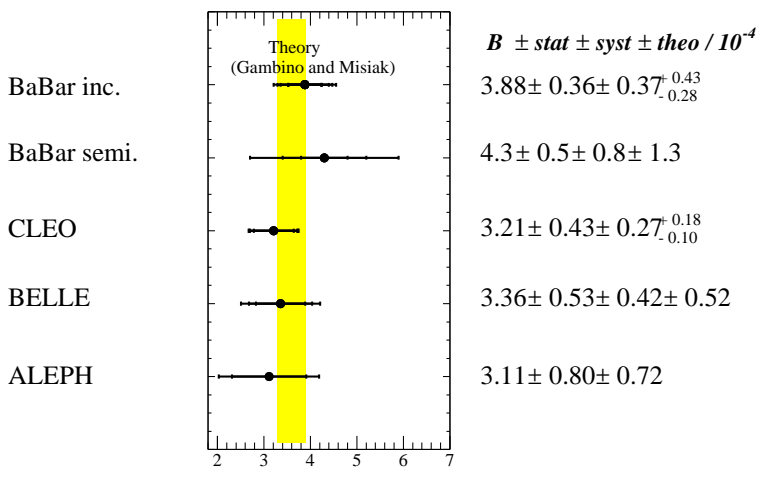

Figure 6. Summary of the individual measurements of the inclusive $b \rightarrow s \gamma$ decays compared with the theoretical predictions.

- they are sensitive to New Physics (heavy particles in the loop);

- the photon energy spectrum can be used to extract non-perturbative QCD parameters, as the b-quark mass and the Fermi motion of the light quark inside the hadrons (which are important to reduce the error on the extraction of $V_{c b}$ and $V_{u b}$ when using inclusive b-semileptonic decay samples);
- the ratio $\frac{B r(b \rightarrow d \gamma)}{B r(b \rightarrow s \gamma)}$ is proportional to the ratio of CKM matrix elements $\left|V_{t d} / V_{t s}\right|^{2}$.

From the theoretical point of view, inclusive decays are "cleaner" than the exclusive ones, because the latter depend upon not yet well controlled form factors. The determination of the CP asymmetry 3 , which is expected to be small in the Standard Model $(<0.5 \%)$, can be a good place for studying non-SM CP violation.

New results from B-factories have been presented. A summary of the exclusive $b \rightarrow s \gamma$ decays is given in Table 5. The measured branching fractions are compatible with the predicted ones and the $\mathrm{CP}$ asymmetry is compatible with zero within the error of about $4 \%$.

First results exist on $b \rightarrow d \gamma$ exclusive decays (involving $\rho^{0}, \rho^{+}$, and $\omega$ ), which combined with the results given in Table imply:

$R=\frac{B r(B \rightarrow \rho \gamma)}{B r\left(B \rightarrow K^{*} \gamma\right)}<0.046$ at $90 \%$ C.L.

This limit is typically a factor two (with a large error) larger than the SM expectations [16], [ 15] (using the current determination of the Unitarity Triangle $\mathrm{R}=0.023 \pm 0.012$ is obtained [ 17]]) and cannot yet be translated into an effective constraint on $\left|V_{t d} / V_{t s}\right|^{2} \propto(1-\bar{\rho})^{2}+\bar{\eta}^{2}$.

\footnotetext{
${ }^{3}$ We recall the definition of the $\mathrm{CP}$ asymmetry: $A_{C P}=$ $\frac{B r(\bar{B} \rightarrow \bar{f})-B r(B \rightarrow f)}{B r(B \rightarrow f)+B r(B \rightarrow f)}$ $\overline{B r(B \rightarrow f)+B r(B \rightarrow f)}$

${ }^{4}$ It can be also reminded that the ratio in equation 4 is cleaner from the theoretical point of view, if only neutral $\mathrm{B}$ mesons are used for the $B \rightarrow \rho \gamma$ decay mode [18]
} 
The BaBar Coll. presented also two new $b \rightarrow$ $s \gamma$ inclusive analyses. The experimental situation, compared with the most recent theoretical calculation [19] is shown in Figure 6. Two comments can be made: on the one hand, the agreement between experimental results and theoretical calculations is excellent, on the other hand the experimental precision is approaching the theoretical uncertainties.

\subsection{Rare leptonic B decays $\left(B \rightarrow X_{s} \ell^{+} \ell^{-}\right)$}

Motivations for the study of rare leptonic and radiative $\mathrm{B}$ decays are similar.

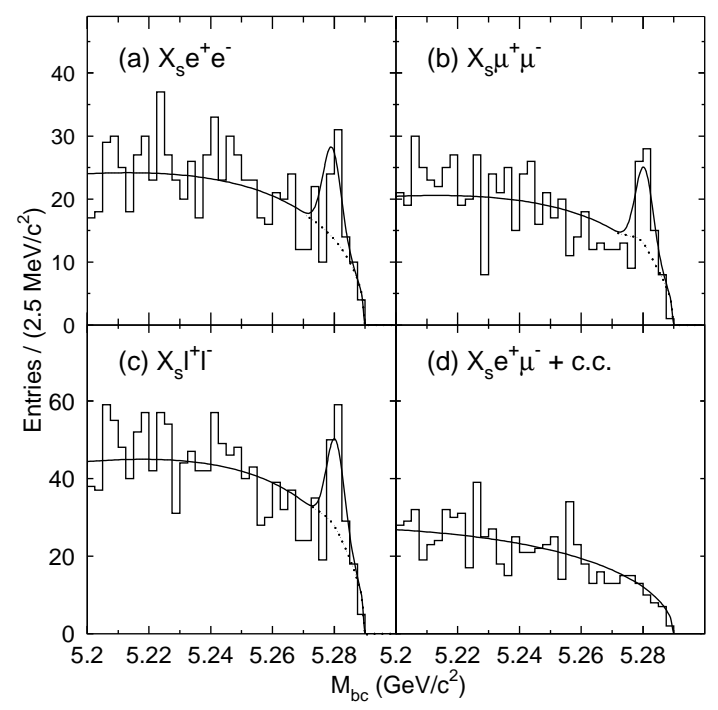

Figure 7. First observation, by Belle Coll. (preliminary result), of the inclusive decay: $b \rightarrow$ $X_{s} \ell^{+} \ell^{-}$. The top left(right) plots show the invariant mass spectrum for the signal in the electron(muon) channels, while the bottom left shows the sum of the two channels. The bottom right plot shows the mass spectrum for the e $\mu$ channel where no signal is expected.

A summary of exclusive leptonic B decay measurements is given in Table 6 .

The Belle Coll. has obtained the first observation of the inclusive $b \rightarrow X_{s} \ell^{+} \ell^{-}$decays (Figure 7 ).
Babar Coll. presented also a limit on the $\operatorname{Br}\left(B^{+} \rightarrow K^{+} \nu \bar{\nu}\right)<9.4 \times 10^{-5}$ at $90 \%$ C.L. ( where the SM expectations is $3.8 \times 10^{-6}$ )

\subsection{B hadronic decays}

Exclusive hadronic B decays are a gold mine for weak and hadronic physics.

One of the important goals for studying these decays is the extraction of the Unitarity Triangle angles.

Hadronic B decays can be schematically classified as:

$\mathrm{B} \rightarrow$ Charmonium decays ;

$\mathrm{B} \rightarrow$ Open Charm decays (DX, DD ,...);

$\mathrm{B} \rightarrow$ Charmless $\mathrm{B}$ decays $(\pi \pi, \mathrm{K} \pi \ldots .$.$) .$

Three kinds of measurement can be performed: branching fractions, $\mathrm{CP}$ asymmetries $\left(A_{C P}\right)$ and time dependent $\mathrm{CP}$ asymmetry $\left(f_{ \pm}=\right.$ $\frac{e^{(-\Delta t / \tau)}}{4 \tau}\left[1 \pm S_{f} \sin \left(\Delta m_{d} \Delta t\right) \mp C_{f} \cos \left(\Delta m_{d} \Delta t\right)\right]$

The cleanest way for extracting a weak angle is the study of the time dependence of CP asymmetry. The "golden channel" is the decay mode: $B \rightarrow J / \Psi K^{0}$ for the extraction of the $\beta$ angle. The angles $\alpha$ and $\gamma$ can be, in principle, extracted from the study of the time dependence in charmless $\mathrm{B}$ decays.

Results from these analyses have been presented in two dedicated plenary talks from the BaBar [ 5] and Belle [6] Coll.. In this paper few examples of experimental results are selected to show the impressive work in this field and also the richness of the hadronic physics informations which can be extracted.

\subsubsection{Open Charm decays}

$B \rightarrow D \pi$ and other colour suppressed decays. The study of Open Charm decays gives an important test for the $\mathrm{B}$ decay dynamics. As an example, $B \rightarrow D \pi$ decay channels can be used.

All the $B \rightarrow D \pi$ decays rates are measured and can be described by the color-allowed and colorsuppressed diagrams (in particular the $B_{d}^{0} \rightarrow$ $\bar{D}^{0} \pi^{0}$ can proceeds only via colour-suppressed diagrams). The amplitudes can be expressed in terms of isospin amplitudes $(\mathrm{I}=1 / 2$ and $\mathrm{I}=3 / 2)$ and of their relative strong phase shift $\left(\delta_{I}=\right.$ $\left.\delta_{I=3 / 2}-\delta_{I=1 / 2}\right)$. Using results from Belle and 
Table 6

Summary of the results on exclusive $B \rightarrow X_{s} \ell^{+} \ell^{-}$decays. Results are still preliminary and averages have been made by the author.

\begin{tabular}{llll} 
Collaboration & $B \rightarrow X_{s} \ell \ell\left[10^{-7}\right]$ & $B \rightarrow K^{*} \ell \ell\left[10^{-7}\right]$ & $B \rightarrow K \ell \ell\left[10^{-7}\right]$ \\
\hline BaBar $(84.4 \mathrm{MB})$ & & $<30$ at $90 \% \mathrm{CL}$ & $7.8_{-2.0-1.8}^{+2.4+1.1}$ \\
Belle $(65.4 \mathrm{MB})$ & $61 \pm 14_{-11}^{+13}$ & $<14$ at 90\%CL & $5.8_{-1.5}^{+1.7} \pm 0.6$ \\
\hline Average & & & $6.4 \pm 1.5$ \\
\hline theory [13] & {$[40-50]$} & {$[10-20]$} & {$[2-5]$}
\end{tabular}

Table 7

Summary of results on colour suppressed modes in Open Charm B decays ( common systematics from $D^{0}$ branching fractions can be neglected at the present level of precision). Part of these results are still preliminary and averages have been made by the author.

\begin{tabular}{lllll} 
Collaboration & $B \rightarrow \bar{D}^{0} \pi^{0}\left[10^{-4}\right]$ & $B \rightarrow \bar{D}^{0} \eta^{0}\left[10^{-4}\right]$ & $B \rightarrow \bar{D}^{0} \omega^{0}\left[10^{-4}\right]$ & $B \rightarrow \bar{D}^{0} \rho^{0}\left[10^{-4}\right]$ \\
\hline CLEO & $2.7_{-0.32}^{+0.36} \pm 0.55$ & - & - & - \\
BaBar ( 50 MB) & $2.9^{-4} \pm 0.3 \pm 0.4$ & $2.4 \pm 0.4 \pm 0.3$ & $2.5 \pm 0.4 \pm 0.3$ & - \\
Belle ( 29 MB) & $3.1 \pm 0.4 \pm 0.5$ & $1.4_{-0.4}^{+0.5} \pm 0.3$ & $1.8 \pm 0.5_{-0.3}^{+0.4}$ & $3.0 \pm 1.3 \pm 0.4(60 \mathrm{MB})$ \\
average & $2.93 \pm 0.34$ & $2.2 \pm 0.4$ & $2.3 \pm 0.4$ & \\
\hline
\end{tabular}

CLEO collaborations it results:

$\cos \delta_{I}=0.866_{-0.036}^{+0.042}$

which is at $3.2 \sigma$ different from unity and indicates sizable final-states re-scattering effects in $D \pi$ decays. Other colour-suppressed modes are now measured and are summarised in Table 7 . The rates are in general twice larger as those expected in the naive factorization approach.

$B \rightarrow D_{s} \pi$ and $B \rightarrow D_{s} K$. The $B \rightarrow D_{s} \pi$ decay mode is expected to proceed via the $b \rightarrow u$ transition, with no penguin contribution and can provide, in principle, a way to determine $\left|V_{u b}\right|$ [ 20]. It seems, nevertheless, difficult to extract $\left|V_{u b}\right|$ with a precision better than $30 \%$. This mode can be used to determine the ratio $R_{\lambda}=\frac{A\left(B^{0} \rightarrow D^{(*)+} \pi^{-}\right)}{A\left(B^{0} \rightarrow D^{(*)}-\pi^{+}\right)}$which is important for a possible extraction of $\sin 2(\beta+\gamma)$ from the study of the time evolution of the $B^{0} \rightarrow D^{(*)-} \pi^{+}$decays. Unfortunately the mode $B^{0} \rightarrow D^{*+} \pi^{-}$ is difficult to access experimentally (background $\left.\bar{B}^{0} \rightarrow D^{*+} \pi^{-}\right)$. The $B \rightarrow D_{s} \pi$ can be then used relating the mode $B^{0} \rightarrow D^{*+} \pi^{-}$to $B^{0} \rightarrow D_{s}^{*+} \pi^{-}$ using $\mathrm{SU}(3)$ symmetry.

The decay $B \rightarrow D_{s} K$ can occur via $\mathrm{W}$-exchange or final state re-scattering and cannot be de-

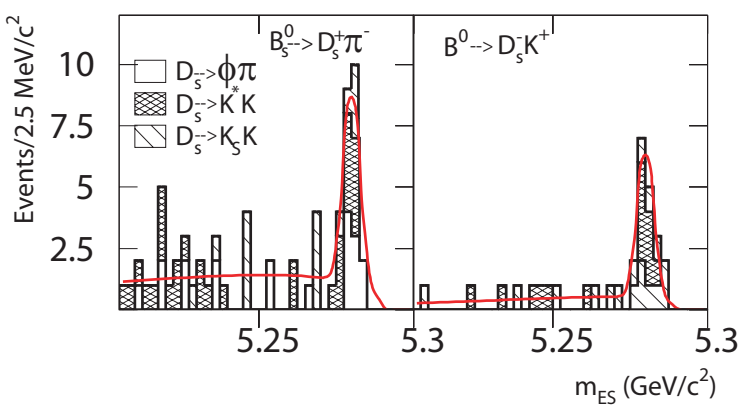

Figure 8. The mass spectrum for the first preliminary observation of the $B \rightarrow D_{s} \pi$ and $B \rightarrow D_{s} K$ decay modes from the BaBar Coll..

scribed by a spectator graph. Wide ranges of prediction of its branching ratio exist (from $3 \times$ $10^{-6}$ to $10^{-4}$ [21]). First results on $B \rightarrow D_{s} \pi$ and $B \rightarrow D_{s} K$ are shown in Figure 8 and summarised in Table 8. The uncertainty on $\operatorname{Br}\left(D_{s} \rightarrow \phi \pi\right)$ is already limiting the precision on the $\operatorname{Br}(B \rightarrow$ $\left.D_{s} \pi\right)$. 
Table 8

Summary of results on $B \rightarrow D_{s} \pi$ and $B \rightarrow D_{s} K$ decay modes. Results are preliminary and averages have been made by the author.

\begin{tabular}{lll} 
Collaboration & $B \rightarrow D_{s} \pi\left[10^{-5}\right]$ & $B \rightarrow D_{s} K\left[10^{-5}\right]$ \\
\hline BaBar( 85 MB) & $3.1 \pm 0.9 \pm 1.0$ & $3.2 \pm 1.0 \pm 1.0$ \\
Belle $(85 \mathrm{MB})$ & $2.4_{-0.8}^{+1.0} \pm 0.7$ & $4.6_{-1.1}^{+1.2} \pm 1.3$ \\
average & $2.7 \pm 0.7 \pm 0.7\left(D_{s} \rightarrow \phi \pi\right)$ & $3.8^{-1.9} \pm 0.9 \pm 1.0\left(D_{s} \rightarrow \phi \pi\right)$ \\
\hline
\end{tabular}

Table 9

Summary of results on the charmless B decays. Many of these results are preliminary and obtained using a registered statistics which depend upon the experiments and the analysed decay modes.

\begin{tabular}{|c|c|c|c|c|c|}
\hline Decay Mode & $\begin{array}{l}\text { Br }\left[10^{-6}\right] \\
(\text { Belle })\end{array}$ & $\begin{array}{l}\mathrm{Br}\left[10^{-6}\right] \\
(\mathrm{BaBar})\end{array}$ & $\begin{array}{l}\mathrm{Br}\left[10^{-6}\right] \\
(\mathrm{CLEO})\end{array}$ & $\begin{array}{l}\mathrm{A}_{C P} \\
\text { (Belle ) }\end{array}$ & $\begin{array}{l}\mathrm{A}_{C P} \\
\text { (BaBar) }\end{array}$ \\
\hline$B^{0} \rightarrow K^{+} \pi^{-}$ & $22.5 \pm 1.9 \pm 1.8$ & $17.9 \pm 0.9 \pm 0.6$ & $17.2_{-2.4}^{+2.5} \pm 1.2$ & $-0.06 \pm 0.09_{-0.02}^{+0.01}$ & $-0.102 \pm 0.050 \pm 0.016$ \\
\hline$B^{0} \rightarrow K^{+} \pi^{0}$ & $13.0_{-2.4}^{+2.5} \pm 1.3$ & $12.8 \pm 1.2 \pm 1.0$ & $11.6_{-2.7-1.3}^{+3.4}$ & $-0.02 \pm 0.19 \pm 0.02$ & $-0.09 \pm 0.09 \pm 0.01$ \\
\hline$B^{0} \rightarrow \pi^{+} \pi^{0}$ & $7.4_{-2.2}^{+2.3} \pm 0.9$ & $5.5 \pm 1.0 \pm 0.6$ & $5.4_{-2.0}^{+2.1} \pm 1.5$ & $0.30 \pm 0.30_{-0.04}^{+0.06}$ & $-0.03 \pm 0.18 \pm 0.02$ \\
\hline$B^{0} \rightarrow K^{0} \pi^{0}$ & $8.0_{-3.3}^{+3.2} \pm 1.6$ & $10.4 \pm 1.5 \pm 0.8$ & $14.6+5.9+2.4$ & & $0.03 \pm 0.36 \pm 0.09$ \\
\hline$B^{0} \rightarrow \pi^{+} \pi^{-}$ & $5.4 \pm 1.2 \pm 0.5$ & $4.6 \pm 0.6 \pm 0.2$ & $4.3_{-1.4}^{+1.6} \pm 0.5$ & $\begin{array}{l}\mathrm{S}=-1.21_{-0.27-0.16}^{+0.38+0.16} \\
\mathrm{C}=0.94_{-0.31}^{+0.25} \pm 0.09\end{array}$ & $\begin{array}{l}\mathrm{S}=0.02 \pm 0.34 \pm 0.05 \\
\mathrm{C}=-0.30 \pm 0.25 \pm 0.04\end{array}$ \\
\hline$B^{0} \rightarrow K^{0} \bar{K}^{0}$ & $<4.1$ & $<7.3$ & $<13$ & & \\
\hline$B^{0} \rightarrow \pi^{0} \pi^{0}$ & $<6.4$ & $<3.6$ & $<5.2$ & & \\
\hline$B^{+} \rightarrow K^{0} \pi^{+}$ & $19.4_{-3.0}^{+3.1} \pm 1.6$ & $17.5 \pm 1.8 \pm 1.3$ & $18.2_{-4.0}^{+4.6} \pm 1.6$ & $0.46 \pm 0.15 \pm 0.02$ & $-0.17 \pm 0.10 \pm 0.02$ \\
\hline$B^{+} \rightarrow K^{+} K^{0}$ & $<2.0$ & $<1.3$ & $<5.1$ & & \\
\hline$B^{+} \rightarrow K^{+} K^{-}$ & $<0.9$ & $<0.6$ & $<1.9$ & & \\
\hline$B^{+} \rightarrow \rho^{0} \pi^{+}$ & $8.0_{-2.0}^{+2.2} \pm 0.7$ & $24 \pm 8 \pm 3$ & $10.4_{-3.4}^{+3.3} \pm 2.1$ & & \\
\hline$B^{0} \rightarrow \rho^{ \pm} \pi^{\mp}$ & $20.8_{-6.3-3.1}^{+6.0+2.8}$ & $28.9 \pm 5.4 \pm 4.3$ & $27.6_{-7.4}^{+8.4} \pm 4.2$ & & $-0.22 \pm 0.08 \pm 0.07$ \\
\hline$B^{0} \rightarrow \rho^{0} \pi^{0}$ & $<5.3^{-0.0-0.1}$ & $<10.6$ & $<5.5$ & & \\
\hline$B^{0} \rightarrow a_{1}^{ \pm} \pi^{\mp}$ & & $6.2_{-2.5}^{+3.0} \pm 1.1$ & & & \\
\hline$B^{+} \rightarrow \rho K$ & $<12$ & $10.7 \pm 1.0_{-1.6}^{+0.9}$ & & & $0.19 \pm 0.14 \pm 0.11$ \\
\hline$B^{+} \rightarrow \eta^{\prime} K^{+}$ & $77.9_{-5.9-8.7}^{+6.2+9.3}$ & $67 \pm 5 \pm 5$ & $80_{-9}^{+10} \pm 7$ & $-0.015 \pm 0.070 \pm 0.009$ & $-0.11 \pm 0.11 \pm 0.02$ \\
\hline$B^{0} \rightarrow \eta^{\prime} K^{0}$ & $68.0_{-9.6-8.2}^{+10.4+8.8}$ & $46 \pm 6 \pm 4$ & $89_{-16}^{+18} \pm 9$ & $0.26 \pm 0.22 \pm 0.03$ & \\
\hline$B^{+} \rightarrow \eta^{\prime} \pi^{+}$ & & $5.4_{-2.6}^{+3.5} \pm 0.8$ & $<12$ & & \\
\hline$B \rightarrow \eta^{\prime} K^{* 0}\left(K^{*+}\right)$ & $<20(90)$ & $<13$ & $<24(35)$ & & \\
\hline$B \rightarrow \eta^{\prime} \rho^{0}$ & $<14$ & & $<12$ & & \\
\hline$B^{+} \rightarrow \eta K^{*+}$ & $26.5_{-7.0}^{+7.8} \pm 1.7$ & $22.1_{-9.2}^{+11.1} \pm 3.3$ & $26.4_{-8.2}^{+9.6} \pm 3.3$ & & \\
\hline$B \rightarrow \eta K^{* 0}$ & $16.5_{-4.2}^{+4.6} \pm 1.2$ & $19.8_{-5.6}^{+6.5} \pm 1.7$ & $13.8_{-4.6}^{+5.4} \pm 1.6$ & & \\
\hline$B \rightarrow \eta K^{+}$ & $5.3_{-1.5}^{+1.8} \pm 0.6$ & & $<6.9$ & & \\
\hline$B \rightarrow \eta \pi^{+}$ & $5.4_{-1.7}^{+2.0} \pm 0.6$ & & $<5.7$ & & \\
\hline$B \rightarrow \eta \rho^{+}\left(\rho^{0}\right)$ & $<2.7(<6.2)$ & & $<15(<10)$ & & \\
\hline$B^{ \pm} \rightarrow \omega \pi^{ \pm}$ & $4.2_{-1.8}^{+2.0} \pm 0.5$ & $6.6_{-1.8}^{+2.1} \pm 0.7$ & $11.3_{-2.9}^{+3.3} \pm 1.4$ & & $-0.01_{-0.31}^{+0.29} \pm 0.03$ \\
\hline$B^{ \pm} \rightarrow \omega K^{ \pm}$ & $9.2_{-2.3}^{+2.6} \pm 1.0$ & $<4$ & $<7.9$ & $-0.21 \pm 0.28 \pm 0.03$ & \\
\hline$B^{0} \rightarrow \omega K^{0}$ & & $5.9_{-1.5}^{+1.7} \pm 0.9$ & $<21$ & & \\
\hline$B^{0} \rightarrow \omega \pi^{0}$ & & $<3.0$ & $<5.5$ & & \\
\hline$B^{ \pm} \rightarrow \phi K^{ \pm}$ & $7.2_{-1.4}^{+1.5} \pm 0.9 \pm 0.4$ & $9.2 \pm 1.0 \pm 0.8$ & $5.5_{-1.8}^{+2.1} \pm 0.6$ & & $-0.05 \pm 0.20 \pm 0.03$ \\
\hline$\overline{B^{ \pm} \rightarrow \phi K^{* \pm}}$ & & $9.7_{-3.4}^{+4.2} \pm 1.7$ & $<22.5$ & & $-0.43_{-0.30}^{+0.36} \pm 0.06$ \\
\hline$B^{0} \rightarrow \phi K^{* 0}$ & & $8.6_{-2.4}^{+2.8} \pm 1.1$ & $11.5_{-3.7-1.7}^{+4.5+1.8}$ & & $0.00 \pm 0.27 \pm 0.03$ \\
\hline$B^{0} \rightarrow \phi K_{S}^{0}$ & $10.0_{-1.7-1.3}^{+1.9+0.9}$ & $8.7_{-1.5}^{+1.7} \pm 0.9$ & $<12.3^{-3.1}$ & $-0.56 \pm 0.41 \pm 0.12$ & \\
\hline$B^{ \pm} \rightarrow \phi \pi^{ \pm}$ & & $<0.56$ & & & \\
\hline$B^{+} \rightarrow \rho^{+} \rho^{0}$ & $38.5 \pm 10.9_{-5.4-7.5}^{+5.9+2.5}$ & & & & \\
\hline
\end{tabular}

\subsubsection{Charmless B decays}

One of the interest of measuring charmless B decays is the determination of the unitarity tri- angle angles $\alpha$ and $\gamma$. In general a given decay mode is described by various tree $(\mathrm{T})$ and penguin $(\mathrm{P})$ diagrams which depend upon weak and 
Table 10

Summary of preliminary results on $\bar{\Lambda}$ and $\lambda_{1}$. The second and third errors correspond, respectively, to the systematic and theoretical uncertainties $\left(\alpha_{s}\right.$ and $\left.1 / m_{b}^{3}\right)$.

\begin{tabular}{lll} 
Collaboration & $\bar{\Lambda}$ & $\lambda_{1}$ \\
\hline BaBar & $0.35 \pm 0.07$ & $-0.17 \pm 0.06 \pm 0.07$ \\
CLEO & $0.39 \pm 0.03 \pm 0.06 \pm 0.12 \mathrm{GeV}$ & $-0.25 \pm 0.02 \pm 0.05 \pm 0.14 \mathrm{GeV}^{2}$ \\
DELPHI & $0.44 \pm 0.04 \pm 0.05 \pm 0.07 \mathrm{GeV}$ & $-0.23 \pm 0.04 \pm 0.05 \pm 0.08 \mathrm{GeV}^{2}$ \\
\hline
\end{tabular}

strong phases. A lot of theoretical investigations have been recently made ([ 22]-[24]). In particular important progress has been made in the last years with the calculation of amplitudes in the heavy quark limit [ 22], but there is still some controversy on the corrections to it [23]. Analyses have been made, essentially on $B \rightarrow P P$ $(\mathrm{P}=$ pseudosclar $)$ with contradictory results.

From the experimental point of view an impressive effort has been made to measure as many branching fractions and $\mathrm{CP}$ asymmetries as possible. Results are given in Table 9. Those on branching fractions are in fairly good agreement among different experiments. CP asymmetries are all compatible with zero. The only "anomaly" is the CP asymmetry in the $\pi^{+} \pi^{-}$channel reported by the Belle Coll..

\section{Determination of $\left|V_{c b}\right|$}

The $\left|V_{c b}\right|$ element of the CKM matrix can be accessed by studying the decay rates of inclusive and exclusive semileptonic $b$-decays.

\subsection{Inclusive analysis.}

The first method to extract $\left|V_{c b}\right|$ makes use of the inclusive semileptonic decays of B-hadrons and of the theoretical calculations done in the framework of the OPE. The inclusive semileptonic width $\Gamma_{\text {s.l. }}$ is expressed as:

$$
\begin{gathered}
\Gamma_{s . l .}=\frac{B R(b \rightarrow c l \nu)}{\tau_{b}}=\gamma_{\text {theory }}\left|V_{c b}\right|^{2} ; \\
\gamma_{\text {theory }}=f\left(\alpha_{s}, m_{b}, \mu_{\pi}^{2}, 1 / m_{b}^{3} \ldots\right) .
\end{gathered}
$$

From the experimental point of view the semileptonic width has been measured by the LEP/SLD and $\Upsilon(4 S)$ experiments with a relative precision of about $2 \%$ :

$\Gamma_{s l}=(0.431 \pm 0.008 \pm 0.007) 10^{-10} \mathrm{MeV} \quad \Upsilon(4 S)$

$$
\Gamma_{s l}=(0.439 \pm 0.010 \pm 0.007) 10^{-10} \mathrm{MeV} \quad \mathrm{Z}^{0}
$$

The average is :

$\Gamma_{s l}=(0.434 \times(1 \pm 0.018)) 10^{-10} \mathrm{MeV}$.

The precision on the determination of $\left|V_{c b}\right|$ is mainly limited by theoretical uncertainties on the parameters entering in the expression of $\gamma_{\text {theory }}$ in equation 6 .

\subsection{Moments analyses}

Moments of the hadronic mass spectrum, of the lepton energy spectrum and of the photon energy in the $b \rightarrow s \gamma$ decay are sensitive to the non perturbative QCD parameters contained in the factor $\gamma_{\text {theory }}$ of equation 6 and in particular to the mass of the $b$ and $c$ quarks and to the Fermi motion of the light quark inside the hadron, $\mu_{\pi}^{2}$. Preliminary results, obtained by BaBar, CLEO and DELPHI Coll., are summarised in Table 10 and in Figure 9. By using the experimental results on $\bar{\Lambda}$ and $\lambda_{1}$ it gives:

$\left|V_{c b}\right|=(40.7 \pm 0.6 \pm 0.8$ (theo.) $) 10^{-3}$ (inclusive)(8)

This result is an important improvement on the determination of the $\left|V_{c b}\right|$ element. Part of the theoretical errors (from $m_{b}$ and $\mu_{\pi}^{2}$ ) is now absorbed in the experimental error and the theoretical error is reduced by a factor two. The remaining theoretical error could be further reduced if the parameters controlling the $1 / m_{b}^{3}$ corrections are extracted directly from experimental data.

\section{3. $B \rightarrow D^{*} \ell \nu$ analysis.}

An alternative method to determine $\left|V_{c b}\right|$ is based on exclusive $\overline{B_{d}^{0}} \rightarrow D^{*+} \ell^{-} \overline{\nu_{l}}$ decays. Us-

\footnotetext{
${ }^{5}$ In another formalism, based on pole quark masses, the $\bar{\Lambda}$ and $\lambda_{1}$ parameters are used, which can be related to the difference between hadron and quark masses and to $\mu_{\pi}^{2}$, respectively.
} 

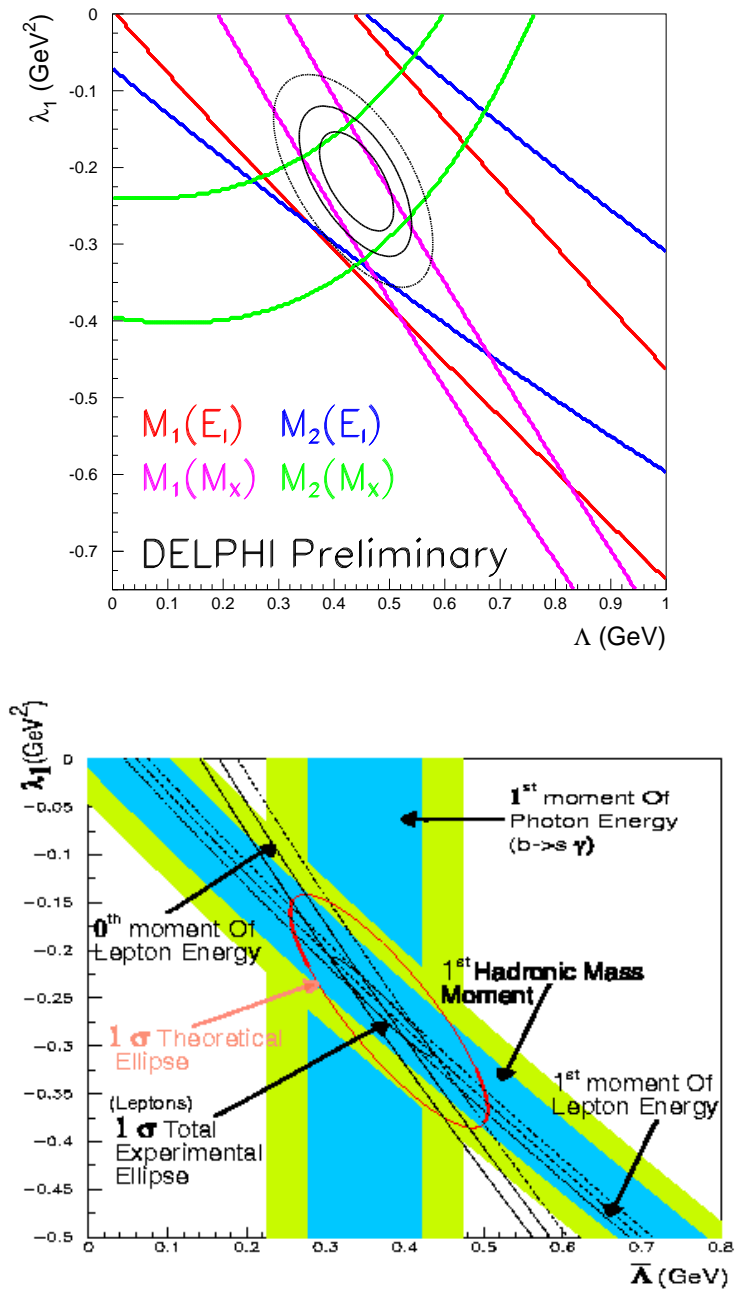

Figure 9. Constraints in the $\bar{\Lambda}-\lambda_{1}$ plane obtained: by the DELPHI Coll. using the measured values of the first two moments of the hadronic mass and lepton energy spectra (top). The bands represent the $1 \sigma$ regions selected by each moment and the ellipses show the 39\%, 68\% and 90\% probability regions of the global fit ; by CLEO Coll. using the first moment of hadronic mass, lepton energy and $\gamma$ energy distributions (bottom). ing HQET an expression for the differential decay rate can be derived

$$
\frac{d \Gamma}{d w}=\frac{G_{F}^{2}}{48 \pi^{2}}\left|V_{c b}^{2} \| F(w)\right|^{2} G(w) ; w=v_{B} \cdot v_{D}
$$

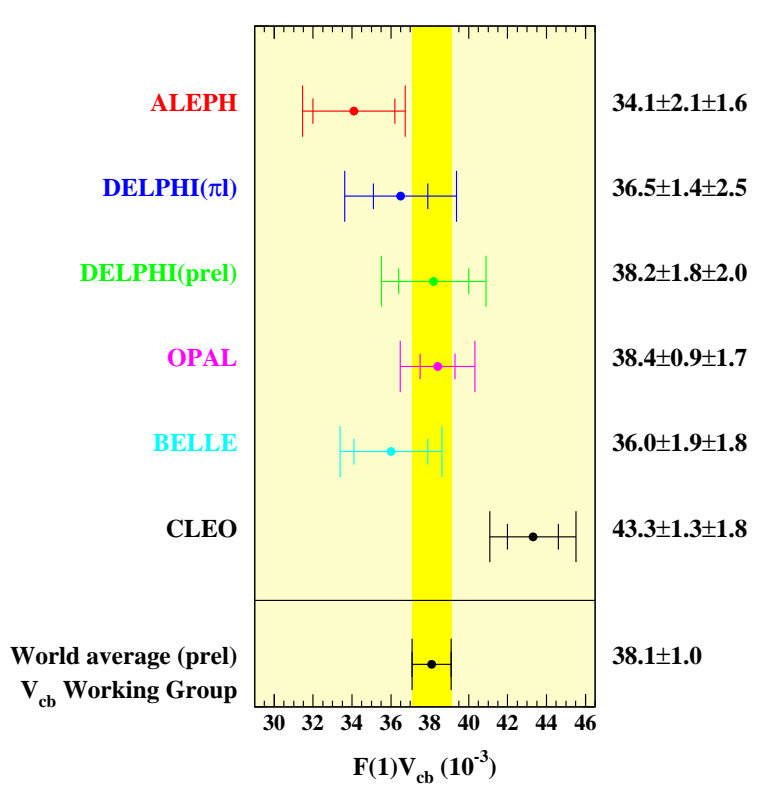

Figure 10. Summary of the measurements of $F(1) \times\left|V_{c b}\right|[25]$.

$w$ is the relative velocity between the $\mathrm{B}\left(v_{B}\right)$ and the D systems $\left(v_{D}\right)$. $\mathrm{G}(w)$ is a kinematical factor and $\mathrm{F}(w)$ is the form factor describing the transition. At zero recoil $(w=1) \mathrm{F}(1)$ goes to unity. The strategy is then to measure $d \Gamma / d w$, to extrapolate at zero recoil and to determine $F(1) \times\left|V_{c b}\right|$.

The experimental results are summarised in Figure 10. Using $\mathrm{F}(1)=0.91 \pm 0.04$ [ 26], it gives [ 25):

$\left|V_{c b}\right|=\left(41.9 \pm 1.1 \pm 1.9(F(1)) 10^{-3}\right.$ (exclusive) $(10)$

Combining the two determinations of $\left|V_{c b}\right|$ (a possible correlation between the two determinations has been neglected) it gives:

$\left|V_{c b}\right|=(40.9 \pm 0.8) 10^{-3}$ (exclusive + inclusive) 


\section{Determination of $\left|V_{u b}\right|$}

This measurement is rather difficult because one has to suppress the large background from the more abundant semileptonic $b$ to $c$ quark transitions.

Several new determinations of the CKM element $\left|V_{u b}\right|$ have been presented at this Conference [27.

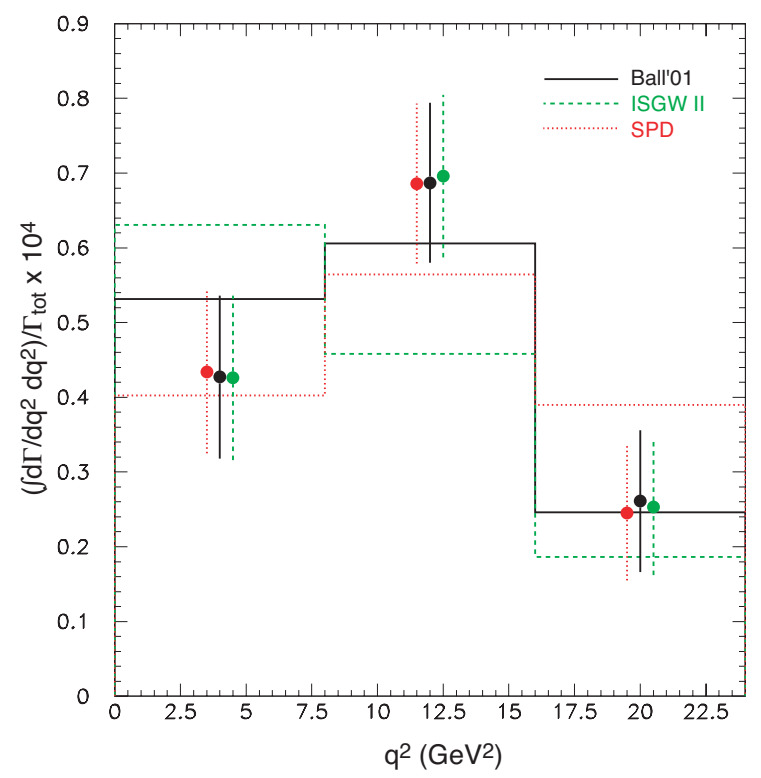

Figure 11. Differential branching fraction for $B^{0} \rightarrow \pi^{-} \ell^{+} \nu$ as a function of $q^{2}$ by the CLEO Coll., compared with the predicted values (histograms) for three models used to extract $\left|V_{u b}\right|$.

\subsection{Determination of $\left|V_{u b}\right|$ using exclusive analyses.}

The first method to determine $\left|V_{u b}\right|$ consists in the reconstruction of the charmless semileptonic B decays: $B \rightarrow \pi(\rho) \ell \nu$.

From the theoretical point of view, the main problem is the determination of the form factors. Light-Cone Sum Rules can provide an evaluation at the 15-20\% level. Lattice QCD calculations

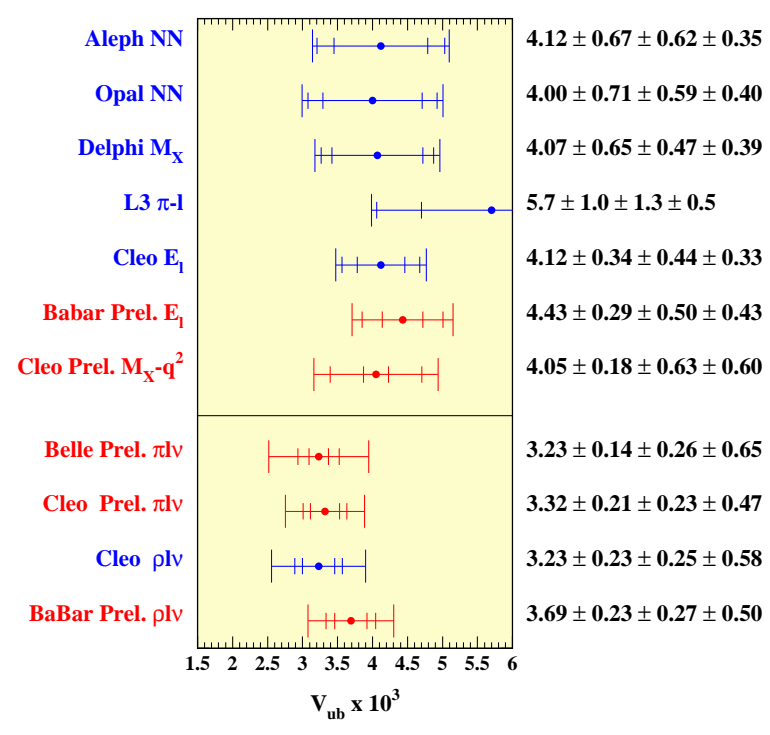

Figure 12. Summary of $\left|V_{u b}\right|$ measurements [27].

give a similar precision but these uncertainties are expected to be reduced in the near future. The main limitation in these calculations is that, at present, they can be used only in the high $q^{2}$ region.

An interesting analysis has been presented by the CLEO Coll. using the $B^{0} \rightarrow \pi^{-} \ell^{+} \nu$ decay mode, extracting the signal rates in three independent regions of $q^{2}$. In this way it is possible to discriminate between models and the fit in Figure 6 shows that the ISGW II model is compatible with data at only $1 \%$ level of probability.

\subsection{Determination of $\left|V_{u b}\right|$ using inclusive analyses.}

As for $\left|V_{c b}\right|$, the extraction of $\left|V_{u b}\right|$ from inclusive semileptonic decays is based on HQET implemented through OPE.

By using kinematical and topological variables, it is possible to select samples enriched in $b \rightarrow u$ transitions. There are, schematically, three main regions in the semileptonic decay phase space:

- the lepton energy end-point region: $E_{\ell}>$ 
$\frac{M_{B}^{2}-M_{D}^{2}}{2 M_{B}}$ (which was at the origin for the first evidence of $b \rightarrow u$ transitions)

- the low hadronic mass region: $M_{X}<M_{D}$ (pioneered by the DELPHI Coll.)

- the high $q^{2}$ region: $M_{\ell \nu}^{2}=q^{2}>\left(M_{B}-\right.$ $\left.M_{D}\right)^{2}$.

The CLEO Coll. has presented an interesting attempt of a combined $M_{X}-q^{2}$ analysis to reduce theoretical uncertainties.

A summary of the different determinations of $\left|V_{u b}\right|$ is given in Figure 12. It is probably too early to make an overall average using all results.

\section{Measurement of $B^{0}-\bar{B}^{0}$ oscillations}
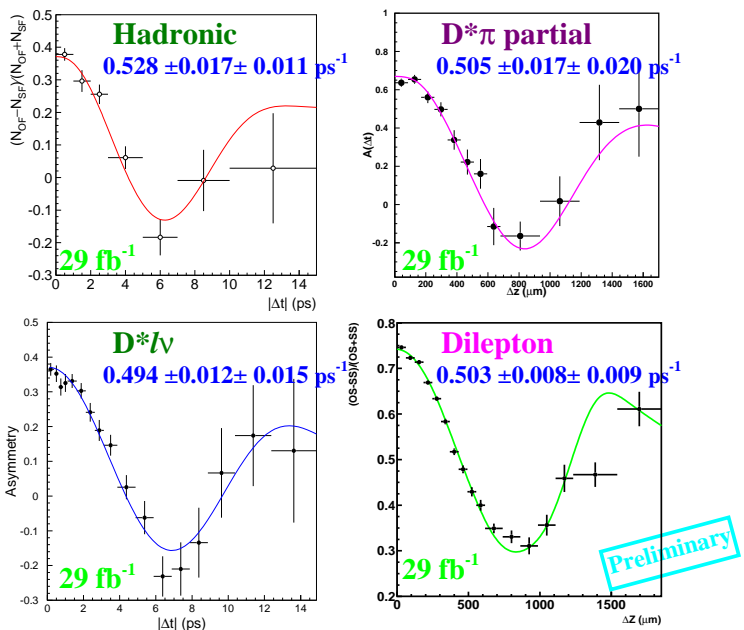

Figure 13. The plots show the $B_{d}^{0}-\bar{B}_{d}^{0}$ oscillations (Belle Coll.). The points with error bars are the data. The result of the fit gives the value for $\Delta m_{d}$.

The probability that a $B^{0}$ meson oscillates into a $\bar{B}^{0}$ or stays as a $B^{0}$ is given by:

$P_{B_{q}^{0} \rightarrow B_{q}^{0}\left(\bar{B}_{q}^{0}\right)}=\frac{1}{2} e^{-t / \tau_{q}}\left(1 \pm \cos \Delta m_{q} t\right)$ where $\tau_{q}$ is the lifetime of the $B_{q}^{0}$ meson, $\Delta m_{q}=$ $m_{B_{1}^{0}}-m_{B_{2}^{0}}$ is the mass difference between the two mass eigenstates . To derive this formula the effects of a $\mathrm{CP}$ violation and of the lifetime difference between the two states have been neglected.

In the Standard Model, $B^{0}-\bar{B}^{0}$ oscillations occur through a second-order process - a box diagram - with a loop of $\mathrm{W}$ and up-type quarks. The box diagram with the exchange of a top quark gives the dominant contribution.

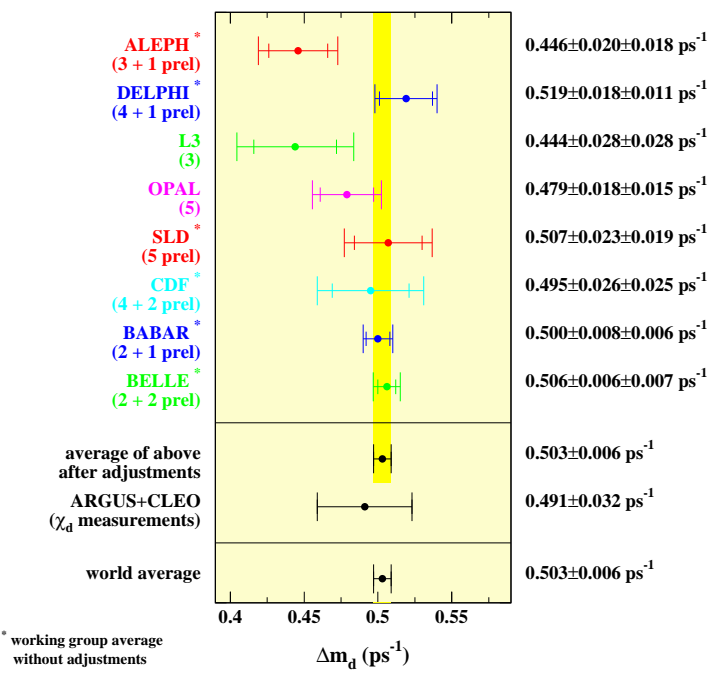

Figure 14. Summary of the measurements of $\Delta m_{d}[28]$.

The oscillation frequency is predicted to be:

$$
\begin{array}{cc}
\Delta m_{d} & \propto V_{c b}^{2} \lambda^{2}\left[(1-\bar{\rho})^{2}+\bar{\eta}^{2}\right] f_{B_{d}}^{2} B_{B_{d}} \\
\Delta m_{s} & \propto V_{c b}^{2} f_{B_{s}}^{2} B_{B_{s}} \\
\frac{\Delta m_{d}}{\Delta m_{s}} & \propto 1 / \xi^{2} \lambda^{2}\left[(1-\bar{\rho})^{2}+\bar{\eta}^{2}\right]
\end{array}
$$

${ }^{6} \Delta m_{q}$ is usually given in $\mathrm{ps}^{-1} .1 \mathrm{ps}^{-1}$ corresponds to 6.58 $10^{-4} \mathrm{eV}$. 
where $\xi=\frac{f_{B_{s}} \sqrt{B_{B_{s}}}}{f_{B_{d}} \sqrt{B_{B_{d}}}}$.

Thus, the measurement of $\Delta m_{d}$ and $\Delta m_{s}$ gives access to the CKM matrix elements $\left|V_{t d}\right|$ and $\left|V_{t s}\right|$ respectively. The difference in the $\lambda$ dependence of these expressions $(\lambda \sim 0.22)$ implies that $\Delta m_{s} \sim 20 \Delta m_{d}$. It is then clear that a very good proper time resolution is needed to measure the $\Delta m_{s}$ parameter. On the other hand the measurement of the ratio $\Delta m_{d} / \Delta m_{s}$ gives the same constraint as $\Delta m_{d}$ but this ratio is expected to have smaller theoretical uncertainties since the ratio $\xi$ is better known than the absolute value of $f_{B} \sqrt{B}_{B}$.

\subsection{Measurement of the $B_{d}^{0}-\bar{B}_{d}^{0}$ oscillation frequency: $\Delta m_{d}$}

The measurement of $\Delta m_{d}$ has been the subject of intense experimental activity in the last ten years. Results are available from the combination of more than 35 analyses, using different event samples, performed by the LEP/SLD/CDF experiments. The combined measurement of $\Delta m_{d}$ have a relative precision of $\sim 2.5 \%$. The new and precise measurements performed at B-Factories are now included, improving the precision by a factor of two.

A typical proper time distribution is shown in Figure 13. The oscillating behaviour is clearly visible. Figure 14 gives the results for $\Delta m_{d}$, obtained by each experiment and the overall average [28]:

$\Delta m_{d}=(0.503 \pm 0.006) p s^{-1}$.

Improvements can still be expected from Bfactories which should reach a few per mille precision on $\Delta m_{d}$.

\subsection{Search for $B_{s}^{0}-\bar{B}_{s}^{0}$ oscillations.}

Since the $B_{s}^{0}$ meson is expected to oscillate more than 20 times faster than the $B_{d}^{0}$ and as $B_{s}$ mesons are less abundantly produced, the search for $B_{s}^{0}-\bar{B}_{s}^{0}$ oscillations is more difficult. The observation of fast oscillations requires the highest resolution on the proper time and on the $B_{s}$ decay length. No signal for $B_{s}^{0}-\bar{B}_{s}^{0}$ oscillations has been observed sofar.

The method used to measure or to put a limit on

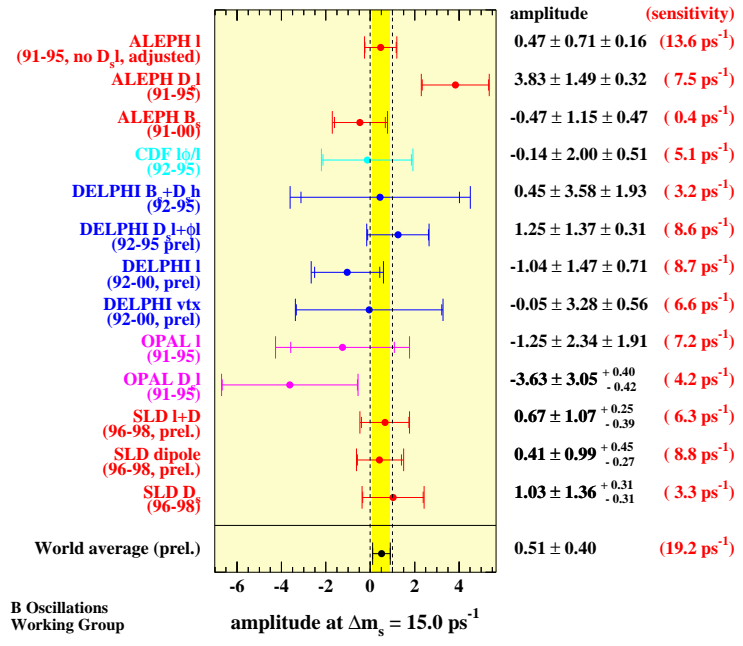

Figure 15. $B_{s}^{0}$ oscillation results. Values of the fitted amplitude at $\Delta m_{s}=15 \mathrm{ps}^{-1}$ and of the sensitivity obtained by each experiment [28].

$\Delta m_{s}$ consists in modifying equation 12 in the following way [29]: $1 \pm \cos \Delta m_{s} t \rightarrow 1 \pm A \cos \Delta m_{s} t$. $\mathrm{A}$ and $\sigma_{A}$ are measured at fixed values of $\Delta m_{s}$ instead of $\Delta m_{s}$ itself. In case of a clear oscillation signal, at a given frequency, the amplitude should be compatible with $\mathrm{A}=1$ at this frequency. With this method it is easy to set a limit. The values of $\Delta m_{s}$ excluded at $95 \%$ C.L. are those satisfying the condition $\mathrm{A}\left(\Delta m_{s}\right)+1.645 \sigma_{A}\left(\Delta m_{s}\right)<1$. Furthermore the sensitivity of the experiment can be defined as the value of $\Delta m_{s}$ corresponding to $1.645 \sigma_{A}\left(\Delta m_{s}\right)=1$ (taking $\mathrm{A}\left(\Delta m_{s}\right)=0$ ), namely supposing that the "true" value of $\Delta m_{s}$ is well above the measurable value.

During the last years impressive improvements in the analysis techniques allowed to increase the sensitivity of this search. Figure 15 gives the details of the different $\Delta m_{s}$ analyses. The combined result of LEP/SLD/CDF analyses [ 28 is shown in Figure 16:

$$
\Delta m_{s}>14.4 \mathrm{ps}^{-1} \text { at } 95 \% \text { C.L. }
$$

The sensitivity is $\quad \Delta m_{s}=19.2 \mathrm{ps}^{-1}$. 


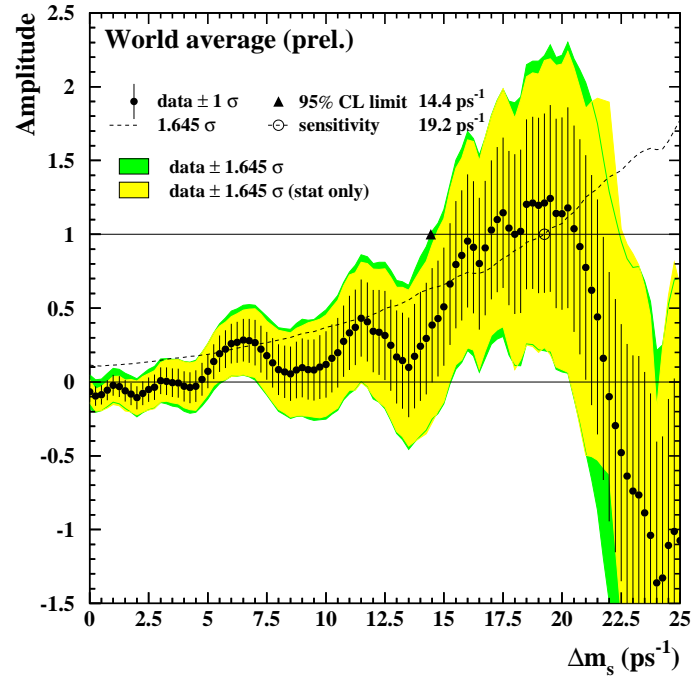

Figure 16. The plot [ 28] shows combined $\Delta m_{s}$ results from LEP/SLD/CDF analyses shown as an amplitude versus $\Delta m_{s}$ plot. The points with error bars are the data; the lines show the $95 \%$ C.L. curves (in dark the systematics have been included). The dotted curve shows the sensitivity.

The present combined limit implies that $B_{s}^{0}$ oscillate at least 30 times faster than $B_{d}^{0}$ mesons. The significance of the "signal" appearing around $17 \mathrm{ps}^{-1}$ is about $2.5 \sigma$ and no claim can be made of the observation of $B_{s}^{0}-\bar{B}_{s}^{0}$ oscillations.

The Tevatron experiments will measure soon $B_{s}^{0}-$ $\bar{B}_{s}^{0}$ oscillations.

\section{Unitarity triangle parameters determi- nation}

Different constraints can be used to select the allowed region for the apex of the triangle in the $\bar{\rho}-\bar{\eta}$ plane. Five have been used sofar: $\epsilon_{k}$, $\left|V_{c b}\right| /\left|V_{c b}\right|, \Delta m_{d}$, the limit on $\Delta m_{s}$ and $\sin 2 \beta$ from the measurement of the $\mathrm{CP}$ asymmetry in the $J / \psi K^{0}$ decays. These constraints are shown in Figure 17.

These measurements provide a set of con-

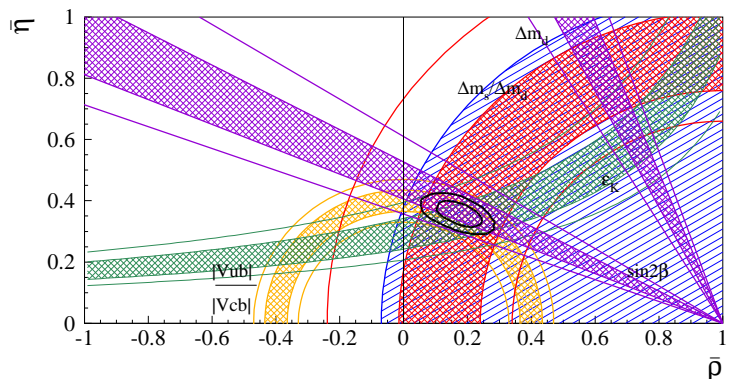

Figure 17. The allowed regions for $\bar{\rho}$ and $\bar{\eta}$ (contours at $68 \%, 95 \%$ ) are compared with the uncertainty bands for $\left|V_{u b}\right| /\left|V_{c b}\right|, \epsilon_{K}, \Delta m_{d}$, the limit on $\Delta m_{s} / \Delta m_{d}$ and $\sin 2 \beta$.

straints which are obtained by comparing measured and expected values of the corresponding quantities, in the framework of the Standard Model (or of any other given model). In practice, theoretical expressions for these constraints involve several additional parameters such as quark masses, decay constants of $\mathrm{B}$ mesons and bagfactors. The values of these parameters are constrained by other measurements (e.g. top quark mass) or using theoretical expectations.

Different statistical methods have been defined to treat the experimental and theoretical errors. The methods essentially differ in the treatment of the latter and can be classified into two main groups: frequentist and Bayesian. The net result is that, if the same inputs are used, the different statistical methods select quite similar values for the different CKM parameters [36]. The results in the following are shown using the Bayesian approach.

Central values and the uncertainties taken for the relevant parameters used in these analyses are given in Table 11 [ 30].

The most crucial test is the comparison between the region selected by the measurements which are sensitive only to the sides of the Unitarity Triangle and the regions selected by the direct measurements of the $\mathrm{CP}$ violation in the kaon $\left(\epsilon_{K}\right)$ or in the $\mathrm{B}(\sin 2 \beta)$ sector. This test is shown in Figure 18 . 
Table 11

Values of the relevant quantities used in the fit of the CKM parameters. In the third and fourth columns the Gaussian and the flat parts of the uncertainty are given, respectively [30]. The values and the errors on $V_{c b}$ are taken from [ 32] and are slightly different with respect to those given in equations 8, 10.

\begin{tabular}{|c|c|c|c|c|}
\hline Parameter & Value & $\begin{array}{l}\text { Gaussian } \\
\sigma\end{array}$ & $\begin{array}{l}\text { Uniform } \\
\text { half-width }\end{array}$ & Ref. \\
\hline $\bar{\lambda}$ & 0.2210 & 0.0020 & - & 31 \\
\hline$\left|V_{c b}\right|$ (excl.) & $42.1 \times 10^{-3}$ & $2.1 \times 10^{-3}$ & - & 32 \\
\hline$\left|V_{c b}\right|$ (incl.) & $40.4 \times 10^{-3}$ & $0.7 \times 10^{-3}$ & $0.8 \times 10^{-3}$ & 32 \\
\hline$\overline{\left|V_{u b}\right|(\text { excl. })}$ & $32.5 \times 10^{-4}$ & $2.9 \times 10^{-4}$ & $5.5 \times 10^{-4}$ & 31 \\
\hline$\left|V_{u b}\right|$ (incl.) & $40.9 \times 10^{-4}$ & $4.6 \times 10^{-4}$ & $3.6 \times 10^{-4}$ & 31 \\
\hline$\Delta m_{d}$ & $0.503 \mathrm{ps}^{-1}$ & $0.006 \mathrm{ps}^{-1}$ & - & 28 \\
\hline$\Delta m_{s}$ & $>14.4 \mathrm{ps}^{-1}$ at $95 \%$ C.L. & sensitivity & $19.2 \mathrm{ps}^{-1}$ & 28 \\
\hline$m_{t}$ & $167 \mathrm{GeV}$ & $5 \mathrm{GeV}$ & - & 34 \\
\hline$f_{B_{d}} \sqrt{\hat{B}_{B_{d}}}$ & $235 \mathrm{MeV}$ & $33 \mathrm{MeV}$ & ${ }_{-24}^{+0} \mathrm{MeV}$ & 33 \\
\hline$\xi=\frac{f_{B_{s}} \sqrt{\hat{B}_{B_{s}}}}{f_{B_{d}} \sqrt{\hat{B}_{B_{d}}}}$ & 1.18 & 0.04 & $\begin{array}{l}+0.12 \\
-0.00\end{array}$ & 33 \\
\hline$\hat{B}_{K}$ & 0.86 & 0.06 & 0.14 & 33 \\
\hline$\overline{\sin 2 \beta}$ & 0.734 & 0.054 & - & 35 \\
\hline
\end{tabular}

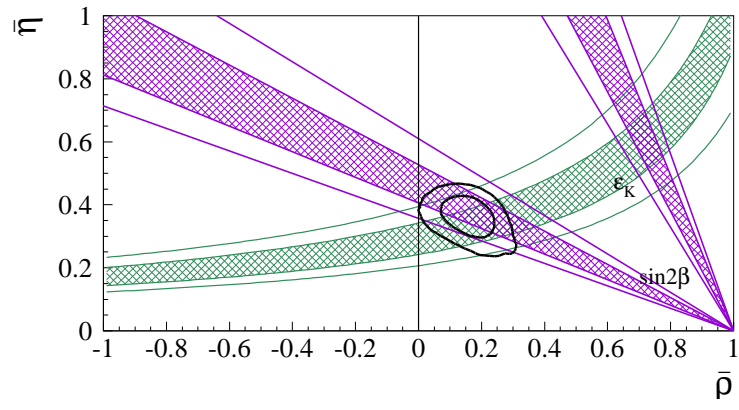

Figure 18. The allowed regions for $\bar{\rho}$ and $\bar{\eta}$ (contours at $68 \%, 95 \%$ ) as selected by the measurement of $\left|V_{u b}\right| /\left|V_{c b}\right|, \Delta m_{d}$, the limit on $\Delta m_{s} / \Delta m_{d}$ as compared with the bands (at 1 and $2 \sigma)$ from $\mathrm{CP}$ violation in the kaon $\left(\epsilon_{K}\right)$ and in the B $(\sin 2 \beta)$ sectors.

It can be translated quantitatively through the comparison between the values of $\sin 2 \beta$ obtained from the measurement of the $\mathrm{CP}$ asymmetry in the $J / \psi K^{0}$ decays and the one determined from "sides" measurements:

$$
\begin{array}{lc}
\sin 2 \beta & =0.725_{-0.065}^{+0.055} \quad \text { sides only } \\
\sin 2 \beta & =0.734 \pm 0.054 \quad \mathrm{~B}^{0} \rightarrow \mathrm{J} / \psi \mathrm{K}^{0} .
\end{array}
$$

The spectacular agreement between these values shows the consistency of the Standard Model in describing the $\mathrm{CP}$ violation phenomena in terms of one single parameter $\eta$. It is also an important test of the OPE, HQET and LQCD theories which have been used to extract the CKM parameters.

Including all five constraints the results are [30]:

$$
\begin{aligned}
& \bar{\eta}=0.357 \pm 0.027 \quad(0.305-0.411) \\
& \bar{\rho}=0.173 \pm 0.046 \quad(0.076-0.260) \\
& \sin 2 \beta=0.725_{-0.031}^{+0.035} \quad(0.660-0.789) \\
& \sin 2 \alpha=-0.09 \pm 0.25 \quad(-0.54-0.40) \\
& \gamma=(63.5 \pm 7.0)^{\circ} \quad(51.0-79.0)^{\circ} \\
& \Delta m_{s}=\left(18.0_{-1.5}^{+1.7}\right) \mathrm{ps}^{-1} \quad(15.4-21.7) \mathrm{ps}^{-1}(17)
\end{aligned}
$$

The ranges within parentheses correspond to $95 \%$ probability.

The results on $\Delta m_{s}$ and $\gamma$ are predictions for those quantities which will be measured in near future. 


\section{Conclusions}

Many and interesting results have been presented at this conference. Traditional main players (LEP/SLD/CLEO) are still delivering results, while the $\mathrm{B}$ factories are moving $\mathrm{B}$ studies into the era of precision physics.

Many quantities have already been measured with a good precision. The lifetimes of the $\mathrm{B}$ and charm hadrons are now measured at the one/few per cent level. $\left|V_{c b}\right|$ is today known with a relative precision better than $2 \%$. In this case, not only, the decay width has been measured, but also some of the non-perturbative QCD parameters entering into its theoretical expression. It is a great experimental achievement and a success of the theory description of the non-perturbative QCD phenomena in the framework of the OPE. Many different methods, more and more reliable, are now available for determining the CKM element $\left|V_{u b}\right|$. The relative precision, today, is about $10 \%$ and will be certainly improved in a near future at the B-factories. The time behaviour of $B^{0}-\bar{B}^{0}$ oscillations has been studied and precisely measured in the $B_{d}^{0}$ sector. The oscillation frequency $\Delta m_{d}$ is known with a precision of about 1\%. $B_{s}^{0}-\bar{B}_{s}^{0}$ oscillations have not been measured sofar, but this search has pushed the experimental limit on the oscillation frequency $\Delta m_{s}$ well beyond any initial prediction. Today we know that $B_{s}^{0}$ oscillate at least 30 times faster than $B_{d}^{0}$ mesons. The frequency of the $B_{s}^{0}-\bar{B}_{s}^{0}$ oscillations will be soon measured at the Tevatron. Nevertheless the impact of the actual limit on $\Delta m_{s}$ for the determination of the unitarity triangle parameters is crucial.

Many B decay branching fractions and relative $\mathrm{CP}$ asymmetries have been measured at the Bfactories. The outstanding result on the determination of $\sin 2 \beta$ has been described in two plenary talks [5],[6]. On the other hand many other exclusive hadronic B rare decays have been measured and constitute a gold mine for weak and hadronic physics, allowing to perform important tests of the $\mathrm{B}$ decay dynamics.

The unitarity triangle parameters are today known with a good precision. A crucial test has been already done: the comparison between the unitarity triangle parameters as determined with quantities sensitive to the sides of the unitarity triangle (semileptonic B decays and oscillations) and with the measurements of $\mathrm{CP}$ violation in the kaon $\left(\epsilon_{K}\right)$ and in the $\mathrm{B}(\sin 2 \beta)$ sectors. The agreement is unfortunately excellent. The Standard Model is "Standardissimo": it is also working in the flavour sector. This agreement is also an important test of the OPE, HQET and LQCD theories which have been used to extract the CKM parameters.

The good news is that all these tests are at best at about $10 \%$ level. The current and the next facilities can surely push these tests to a $1 \%$ accuracy. It is important to note that charm physics can play an important role in this respect (providing a laboratory for LQCD) and the Charm-factory (CLEO-C) will play a central role for these issues.

\section{Acknowledgements}

I would like to thank the organisers for the invitation and for having set up a very interesting conference in a nice atmosphere.

I really would like to thank many colleagues from Babar, Belle, CLEO, CDF, LEP, FOCUS, SELEX and SLD experiments, which help me in the preparation of the talk. I would also like to remember the important work made from the members of the Heavy Flavour Working Groups who prepared a large fraction of the averages quoted in this note. They are all warmly thanked.

Finally, special thanks to E. Barberio, M. Battaglia, P. Kluit, P. Roudeau and F. Parodi both for the help and the support during the preparation of the talk and for the careful reading of these proceedings.

\section{REFERENCES}

1. see Y. Nir, these proceedings.

2. N. Cabibbo, Phys. Rev. Lett. 10 (1963) 531.

3. M. Kobayashi and K. Maskawa, Prog. Theor. Phys. 49 (1973) 652.

4. A.J. Buras, M.E. Lautenbacher and G. Ostermaier, Phys. Rev. D50 (1994) 3433.

5. see Y. Karyotakis (BaBar Coll.), these proceedings. 
6. see M. Yamauchi (Belle Coll.), these proceedings.

7. P. Abreu et al. (DELPHI Coll.), Phys. Lett. B345 (1995) 598.

R. Akers et al. (OPAL Coll.), Zeit. Phys. C66 (1995) 19.

D. Buskulic et al. (ALEPH Coll.), Zeit. Phys. C69 (1996) 393.

8. R. Akers et al. (OPAL Coll.), Zeit. Phys. C66 (1995) 19.

M. Feindt et al. (DELPHI Coll.), Internal Note DELPHI 95-105 PHYS 540, submitted paper to EPS-HEP95.

9. M. Feindt et al. (DELPHI Coll.), Internal Note DELPHI 95-107 PHYS 542, submitted paper to EPS-HEP95.

10. LEP B Lifetime Working Group: http: \\lepbosc.web.cern.ch/LEPBOSC/lifetimes/ lepblife.html

11. E.Franco, V.Lubicz, F. Mescia, C.Tarantino, Nucl.Phys. B633 (2002) 212. (hep$\mathrm{ph} / 02030890)$.

12. R. Ammar et al. (CLEO Coll.), Phys. Rev. Lett. 71 (1993) 674.

13. A. Ali, E. Lunghi, G. Greub and G. Hiller Phys. Rev. D66 (2002) 034002 (hep$\mathrm{ph} / 0112300$ ).

14. M. Beneke, T. Felmann and D. Seidel, Nucl. Phys. B612 (2001) 25 (hep-ph/0106067).

15. S. W. Bosch and G. Buchalla Nucl. Phys. B621 (2002) 459 (hep-ph/0106081).

S. W. Bosch hep-ph/0208203 , hep$\mathrm{ph} / 0208204$.

16. A. Ali and A. Ya. Parkhomenko, Eur. Phys. J C23 (2002) 89. (hep-ph/0105302).

17. A. Ali, E. Lunghi hep-ph/0206242.

K. Kiers, A. Soni, G.-H. Wu, Phys. Rev. D62 (2000) 116004. (hep-ph/0006280)

18. D. Atwood, B. Blok, A. Soni, Int. J. Mod. Phys. A11 (1996) 3743 ; Nuovo Cim. 109A (1996) 873.

19. P. Gambino and N. Misiak, Nucl. Phys. B611 (2001) 338 (hep-ph/0104034).

20. D. Choudhury et al., Phys. Rev D 45 (1992) 217. ; C.S. Kim et al., Phys. Rev D 63 (2001) 094506.

21. D. Du, Phys. Lett. B 406 (1997) 110.

B. Block et al., Phys. Rev. Lett. 78 (1997)
3999.

K. Ukai, Proc. of BCP4 , Feb. 2001, IseShima, Japan (pag. 287).

C.-K. Chua, W.-S. Hou, K.-C. Yang, Phys. Rev D 65 (2002) 096007.

22. M. Beneke, G. Buchalla, M. Neubert, C.T. Sachrajda, Phys.Rev.Lett. 83 (1999) 1914-1917; Nucl.Phys. B591 (2000) 313-418; Nucl.Phys. B606 (2001) 245-321.

23. M. Ciuchini, E. Franco, G. Martinelli, M. Pierini and L. Silvestrini, AIP Conf.Proc. 602 (2001) 180-193; Phys. Lett B515 (2001) 33.

24. see for istance: M. Gronau, J. L. Rosner, Phys.Rev. D66 (2002) 053003; Phys.Rev. D65 (2002) 093012; Phys.Rev. D65 (2002) 013004; Erratum-ibid. D65 (2002) 079901

C.-W. Chiang, J. L. Rosner, Phys.Rev. D65 (2002) 074035.

R. Fleischer and J. Matias, Phys. Rev. D 66 (2002) 054009 (hep-ph/0204101).

A.J. Buras and R. Fleischer, Eur. Phys. J. C16 (2000) 97 (hep-ph/0003323).

25. $V_{c b}$ Working Group: http: \\lepvcb.web.cern.ch/LEPVCB/

26. see L. Lellouch, these proceedings; A. S. Kronfeld, P. B. Mackenzie, J. N. Simone, S. Hashimoto, S. M. Ryan, proceedings of FPCP, May 16-18, Philadelphia, Pennsylvania (hep-ph/0110253); Phys.Rev. D66 (2002) 014503.

27. see M. Battaglia, these proceedings.

28. LEP Working group on oscillations:

http: \\lepbosc.web.cern.ch/LEPBOSC/

combined_results/amsterdam_2002/.

29. H.G. Moser and .A. Roussarie, Nucl. Instum. Meth. A384 (1997) 491.

30. see F. Parodi, these proceedings.

31. Results presented at the CKM Unitarity Triangle Workshop, CERN Feb 2002:

http: \\ckm-workshop.web.cern.ch/ckm-

workshop/ in particular see also:

LEP Working group on $\left|V_{c b}\right|$ : http: \\lepvcb.web.cern.ch/LEPVCB/ Winter 2002 averages.

LEP Working group on $\left|V_{u b}\right|$ :

http: \\battagl.home.cern.ch/battagl/vub/vub.html.

32. M. Artuso and E. Barberio, hep-ph/0205163.

33. see talk of L. Lellouch, these proceedings; 
34. F. Abe et al., CDF Collaboration, Phys. Rev. Lett. 74 (1995) 2626.

S. Abachi et al., D0 Collaboration, Phys. Rev. Lett. 74 (1995) 2632.

35. Average from $\mathrm{Y}$. Nir see these proceedings based on: R. Barate et al. (ALEPH Collaboration) Phys. Lett. B492 (2000), 259274; K. Ackerstaff et al. (OPAL Collaboration) Eur. Phys. C5 (1998) 379; T. Affolder at al. Phys. Rev. D61 (2000) 072005; B. Aubert et al. (Babar Collaboration) hepex/0207042; K. Abe at al. (Belle Collaboration) hep-ex/0207098.

36. Results presented at the CKM Unitarity Triangle Workshop, CERN Feb 2002. http: \\ckm-workshop.web.cern.ch/ckmworkshop/

For the description of the methods see: M. Ciuchini et al., JHEP 0107 (2001) 013 (hep-ph/0012308).

A. Höcker, H. Lacker, S. Laplace, F. Le Diberder, Eur. Phys. J. C21, 225 (2001).

G. P. Dubois-Felsmann D. G. Hitlin, F. C. Porter and G. Eigen, CALT 68-2396 June2002.

D. Atwood and A. Soni, Phys.Lett. B508 (2001) 17. (hep-ph/0103197) 